Communication

\title{
Selective Halogen-Lithium Exchange of 1,2-Dihaloarenes for Successive [2+4] Cycloadditions of Arynes and Isobenzofurans
}

\author{
Shohei Eda and Toshiyuki Hamura * \\ Department of Applied Chemistry for Environment, School of Science and Technology, \\ Kwansei Gakuin University, 2-1 Gakuen, Sanda, Hyogo 669-1337, Japan; \\ E-Mail: shoheie816@gmail.com \\ * Author to whom correspondence should be addressed; E-Mail: thamura@kwansei.ac.jp; \\ Tel./Fax: +81-79-565-7591.
}

Academic Editor: Hiroto Yoshida

Received: 1 October 2015 / Accepted: 20 October 2015 / Published: 23 October 2015

\begin{abstract}
Successive [2+4] cycloadditions of arynes and isobenzofurans by site-selective halogen-lithium exchange of 1,2-dihaloarenes were developed, allowing the rapid construction of polycyclic compounds which serve as a useful synthetic intermediates for the preparation of various polyacene derivatives.
\end{abstract}

Keywords: aryne; isobenzofuran; [2+4] cycloaddition; 1,2-dihaloarenes; polyacene; halogen-lithium exchange

\section{Introduction}

We previously reported dual annulations of dibromoisobenzofuran 1, a formal equivalent of didehydroisobenzofuran A, via [2+4] cycloadditions of aryne [1-9] and isobenzofuran [10-23] (Scheme 1). Selective bromine-lithium exchange from the starting two dibromides $\mathbf{2}$ and $\mathbf{3}$ enables the tandem generation of arynes and dual cycloadditions with two different arynophiles (step 1 and step 2). Importantly, successive process can be performed in one-pot by sequential addition of the arynophiles, affording various functionalized polycyclic aromatic compounds [24-26]. 


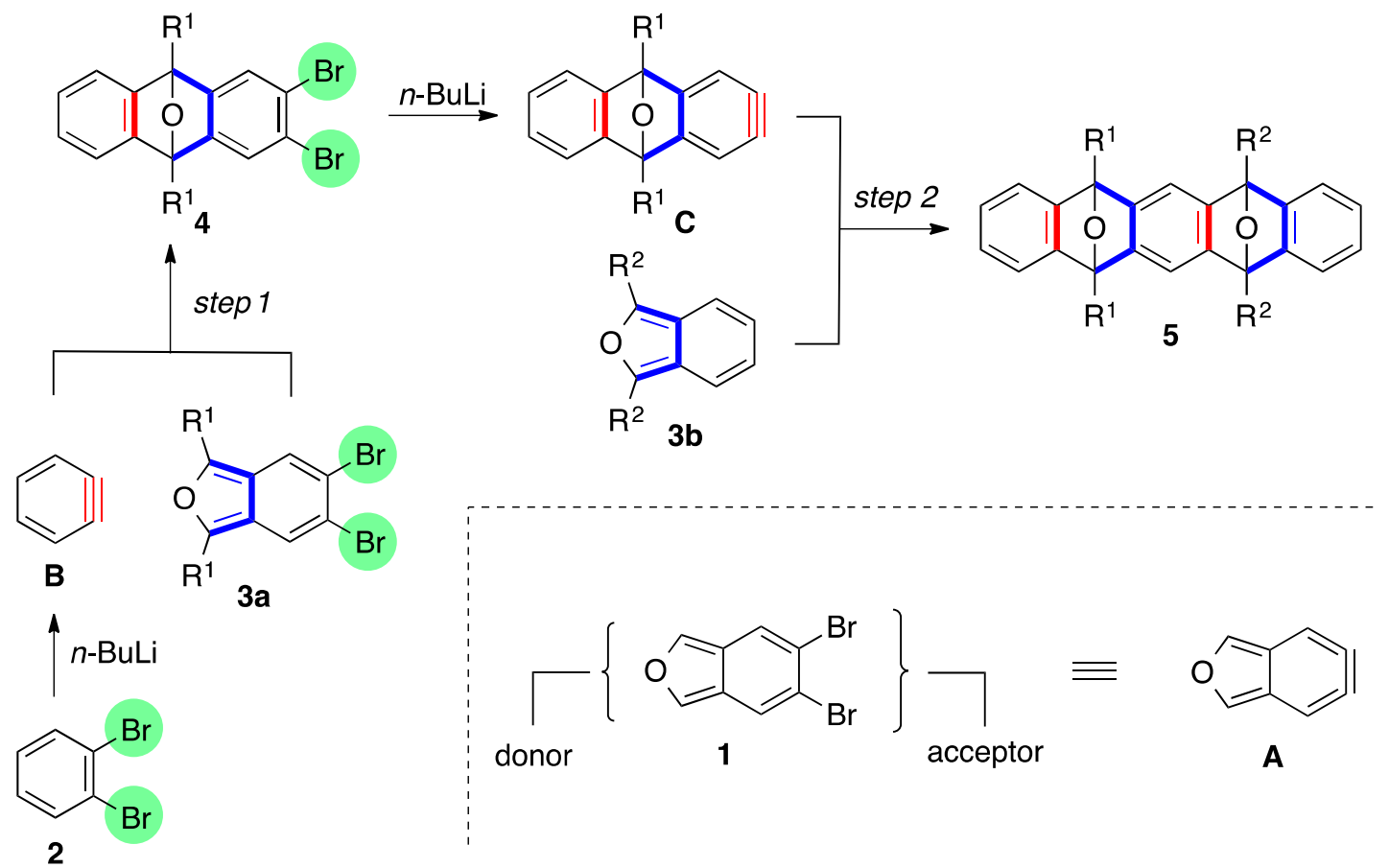

Scheme 1. Successive [2+4] cycloadditions of arynes and isobenzofurans.

This sequential cycloaddition, however, has a limitation in that the introduction of electron withdrawing groups on the benzene ring in aryne precursor (e.g., 2b) is required to restrict the competitive formation of the dual cycloadduct (Scheme 2). In fact, treatment of dibromobenzene $\mathbf{2 a}$ with $n$-BuLi in the presence of dibromoisobenzofuran 1 gave cycloadduct $\mathbf{6 a}$ in $18 \%$ yield, accompanied by a sizable amount of bis-cycloadduct $7 \mathbf{a}(25 \%)$. This result indicates that in addition to the generation of benzyne $\mathbf{B}$, similar reactivity of two dibromides $\mathbf{2} \mathbf{a}$ and $\mathbf{6 a}$ with $n$-BuLi caused the competitive generation of aryne $\mathbf{D}$ from the initially formed cycloadduct 6a. In this case, excess amounts of the starting material $2 \mathbf{a}$ (5.0 equiv.) improved the yields of the mono-cycloadduct $6 \mathbf{a}(42 \%)$ by selective generation of benzyne $\mathbf{B}$. However, it is not an essential solution, since existing of the large amount of the starting material $\mathbf{2 a}$ disturbed the second [2+4] cycloaddition with $\mathbf{6 a}$ in a one-pot process.

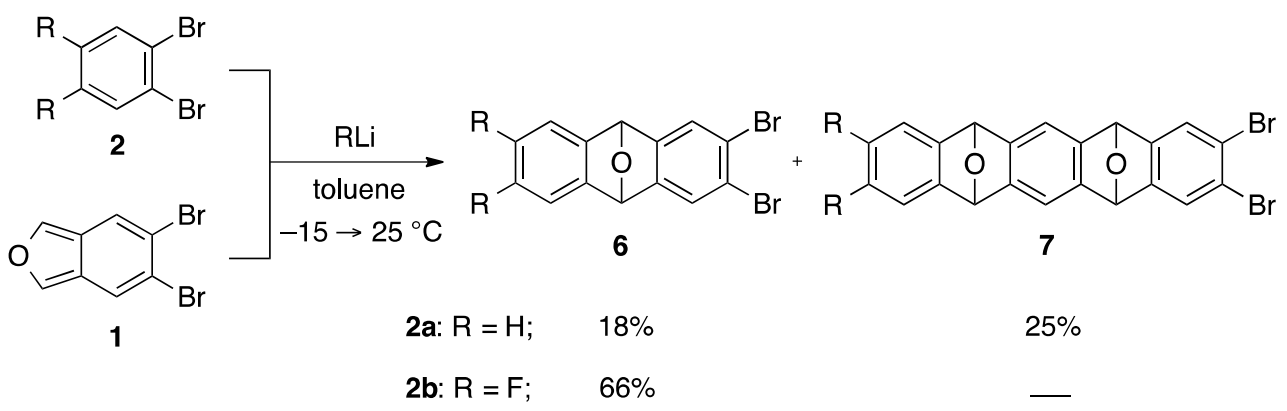<smiles>c1ccccc1</smiles>

B

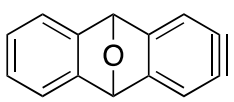

D

Scheme 2. Previous study on the [2+4] cycloaddition of benzyne and dibromoisobenzofuran.

To expand the synthetic utility of this successive processes, we reexamined [2+4] cycloadditions of aryne and isobenzofuran including the parent benzyne species $\mathbf{B}$ as an initial cycloaddition (vide supra). The key to achieve this sequential process is search for a suitable aryne precursor to enable the selective halogen-lithium exchange [27-30]. Along these lines, we select 1,2-dihaloarenes as an aryne precursor 
and expect that controlling the reactivity of the halogen would be possible by taking advantage of the following two features: (1) utilization of the more electropositive halogen (type 1); or (2) tuning the reactivity of halogen by the adjacent halogen (type 2) as shown in Scheme 3. The naive idea of the second strategy is that the strong electron-withdrawing ability of the adjacent halogen might reinforce the electrophilicity of the halogen atom, thus facilitating the halogen-lithium exchange. Importantly, these two factors would allow for the site-selective halogen-lithium exchange among three halides, i.e., dihaloarene, dihaloisobenzofuran, and dihalocycloadduct (Scheme 1), which leads to the tandem generation of arynes and multiple cycloadditions with two or three different arynophiles. We report herein the positive resolution of this scenario [31,32].

1) Type 1: Utilization of more electropositive halogen

2) Type 2: Tuning the reactivity by adjacent halogen
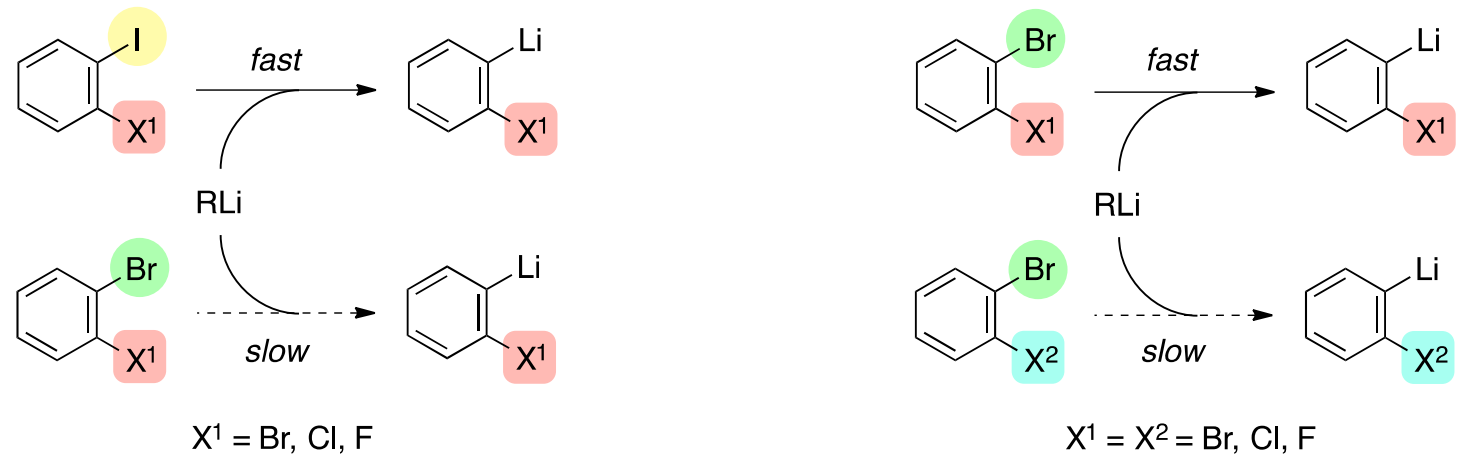

Scheme 3. Two strategies for selective halogen-lithium exchange of 1,2-dihaloarenes.

\section{Results and Discussion}

Table 1 shows initial model reaction for selective generation of benzyne species B. Upon treatment of 1-bromo-2-iodobenzene (8a) with 1.2 equiv. of $n$-BuLi in the presence of 1.0 equiv of 5,6-dibromoisobenzofuran (1) in toluene at $-78^{\circ} \mathrm{C}$, iodine-lithium exchange of 8 a occurred cleanly. The aryllithium intermediate, thus formed, underwent 1,2-elimination of $\mathrm{LiBr}$ to generate benzyne $\mathbf{B}$, which was trapped with $\mathbf{1}$ to give mono-cycloadduct $\mathbf{6 a}$ in $60 \%$ yield (entry 1 ). It is clear that the formation of the bis-cycloadduct 7a via the bromine-lithium exchange of 6a was not fully but mainly suppressed (9\%) in comparison with the corresponding reaction of dibromide $\mathbf{2 a}$ used as a benzyne source. Same reaction at higher reaction temperature $\left(-15 \rightarrow 25^{\circ} \mathrm{C}\right)$ gave a better yield of the desired product $6 \mathbf{6}(78 \%)$, and the bis-cycloadduct $7 \mathbf{a}$ was obtained only in $1 \%$ yield (entry 2). Using 1-chloro-2-iodobenzene (8b) as a benzyne precursor again proved feasible with $n$-BuLi (toluene, $-15 \rightarrow 25^{\circ} \mathrm{C}$ ), affording $6 \mathrm{a}$ in $62 \%$ yield (entry 4). Moreover, the corresponding reaction of iodide $8 \mathbf{c}$ having a fluorine atom at 2-position as a leaving group gave moderate yield of 6a (entries 5-6). These results indicate that halogen-lithium exchange selectively occurred at the more electropositive iodine atom in iodo-halides 8a-8c (Type 1 in Scheme 3), smoothly generating (2-halo)phenyllithiums, respectively, whereas the dibromoisobenzofuran $\mathbf{1}$ and the dibromocycloadduct 6a almost untouched under these conditions [33-36]. As for the moderate yield of the cycloadduct $6 \mathbf{a}$ in the reaction of the dihalides $8 \mathbf{b}$ and $8 \mathrm{c}$, the lower leaving ability of halogen $(\mathrm{Cl}$ and $\mathrm{F})$ in comparison with bromine in aryllithium species would affect the elimination of lithium halide and subsequent generation of benzyne $\mathbf{B}$ [37]. Based on these reaction outcomes, it is safe to say 
that use of 1,2-dihaloarenes $\mathbf{8 a - 8 c}$ possessing a more electropositive iodine atom is favored as a benzyne precursor over the bromide $\mathbf{2} \mathbf{a}$ in terms of selectivity and yield.

Table 1. Initial model study.

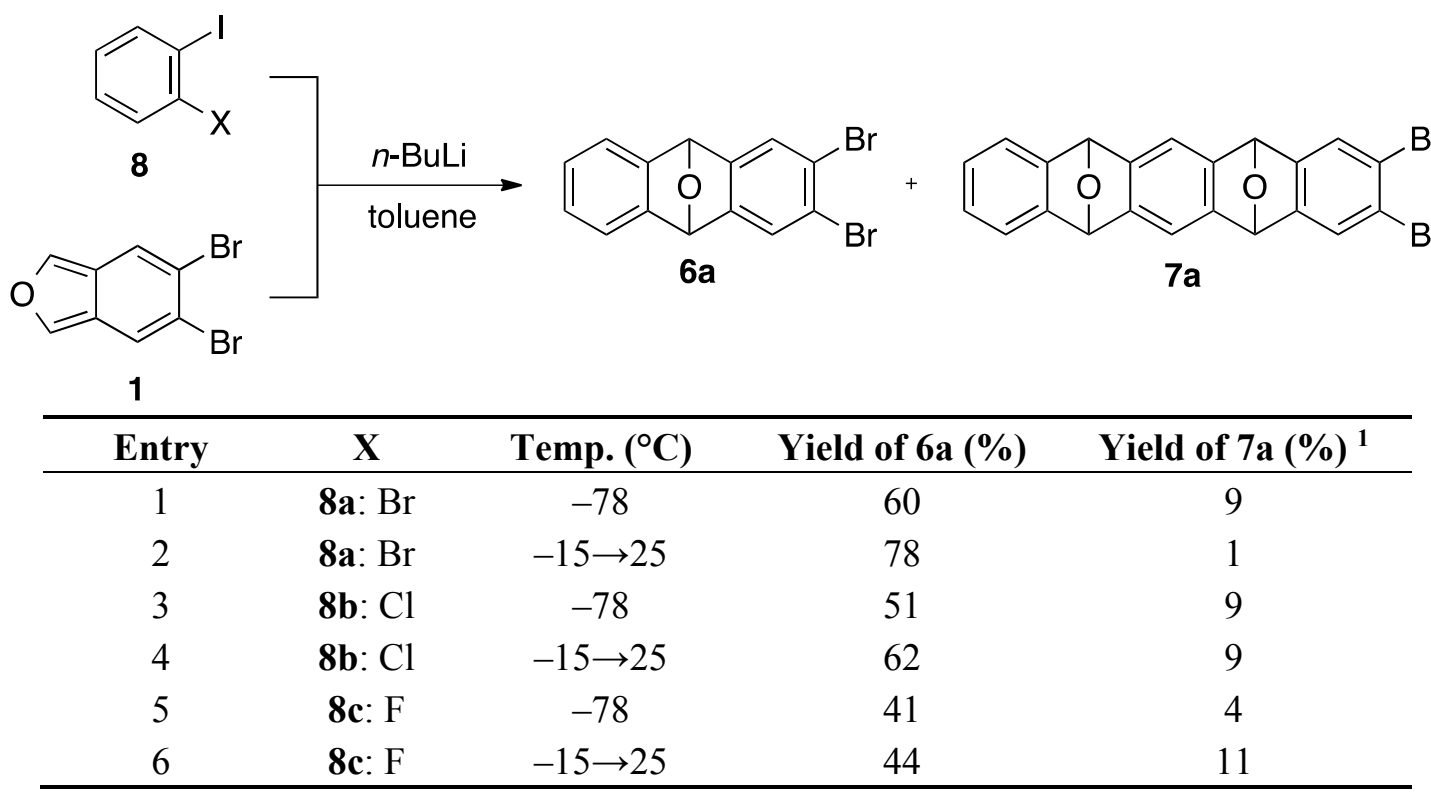

${ }^{1}$ The cycloadduct $7 \mathbf{a}$ was obtained as a mixture of disastereomers (ds: 44/56 58/42).

Further study revealed that 5,6-dibromo-1,3-diphenylisobenzofuran (9a) was also a suitable reactive partner, which cyclized with benzyne $\mathbf{B}$, generated by treatment of iodobromide 8a with $n$-BuLi (toluene, $-15 \rightarrow 25^{\circ} \mathrm{C}$ ), affording substituted epoxyanthracene $\mathbf{1 0}$ in $72 \%$ yield (Scheme 4 ).

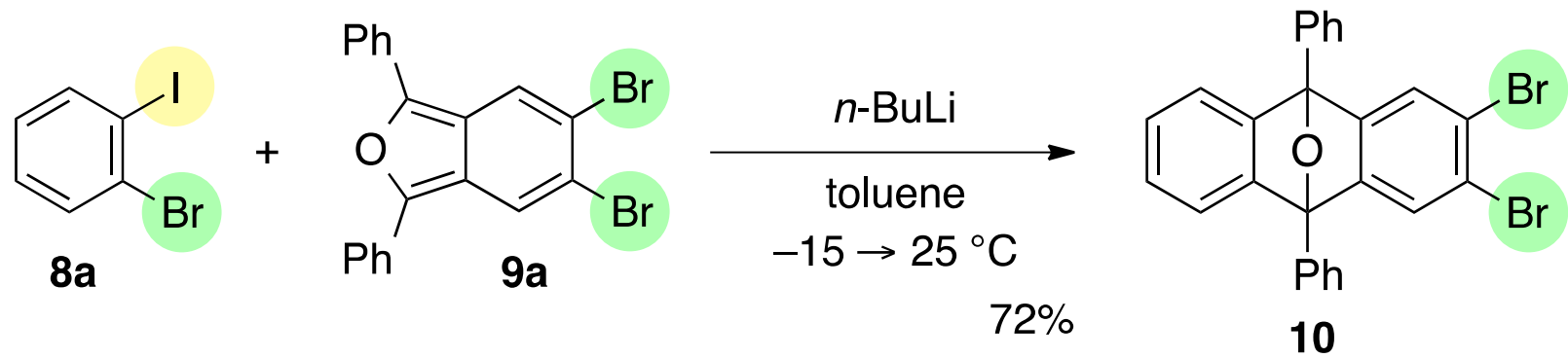

Scheme 4. $[2+4]$ cycloaddition of benzyne $\mathbf{B}$ and isobenzofuran 9a.

We next examined second [2+4] cycloaddition of aryne generated from the first cycloadduct. To explore another mode of selective halogen-lithium exchange of 1,2-haloarenes, i.e., reactivity control by adjacent halogen (type 2 in Scheme 3) [38,39], two different halogens were introduced to isobenzofuran. Upon treatment of dibromide $\mathbf{1 0}$ with 1.3 equiv. of $n$-BuLi in the presence of 1.1 equiv. of 5-bromo-6-chloro-1,3-diphenylisobenzofuran (9b) [40] (toluene, $25{ }^{\circ} \mathrm{C}$ ), aryne $\mathbf{E}$ was selectively generated and subsequent trapping with $\mathbf{9 b}$ gave mono-cycloadduct $\mathbf{1 1}$ in 54\% yield as a mixture of diastereomers (Scheme 5). In this case, bis-cycloadduct 12, caused by the generation of aryne $\mathbf{F}$, was produced in $16 \%$ yield. This observed site-selectivity in the bromine-lithium exchange among three bromides 9b, 10, and 11 was unexpected, because (2-chlorophenyl)lithium 14 was more thermodynamically stable than (2-bromophenyl)lithium 15 by existing of a more electron withdrawing chlorine atom, which would 
suggest the favorable formation of aryne $\mathbf{F}$ over that of aryne $\mathbf{E}$ [41]. Aside from the unanticipated site-selectivity in this bromine-lithium exchange, further introduction of fused ring onto the dual cycloadduct 11 was realized by the third [2+4] cycloaddition of aryne $\mathbf{F}$ and isobenzofuran $9 \mathbf{c}$ by treatment of 11 with $n$-BuLi under the similar conditions, affording polycyclic compound $\mathbf{1 3}$ in $66 \%$ yield, which is expected to be suitably converted to substituted heptacenes [42-45].
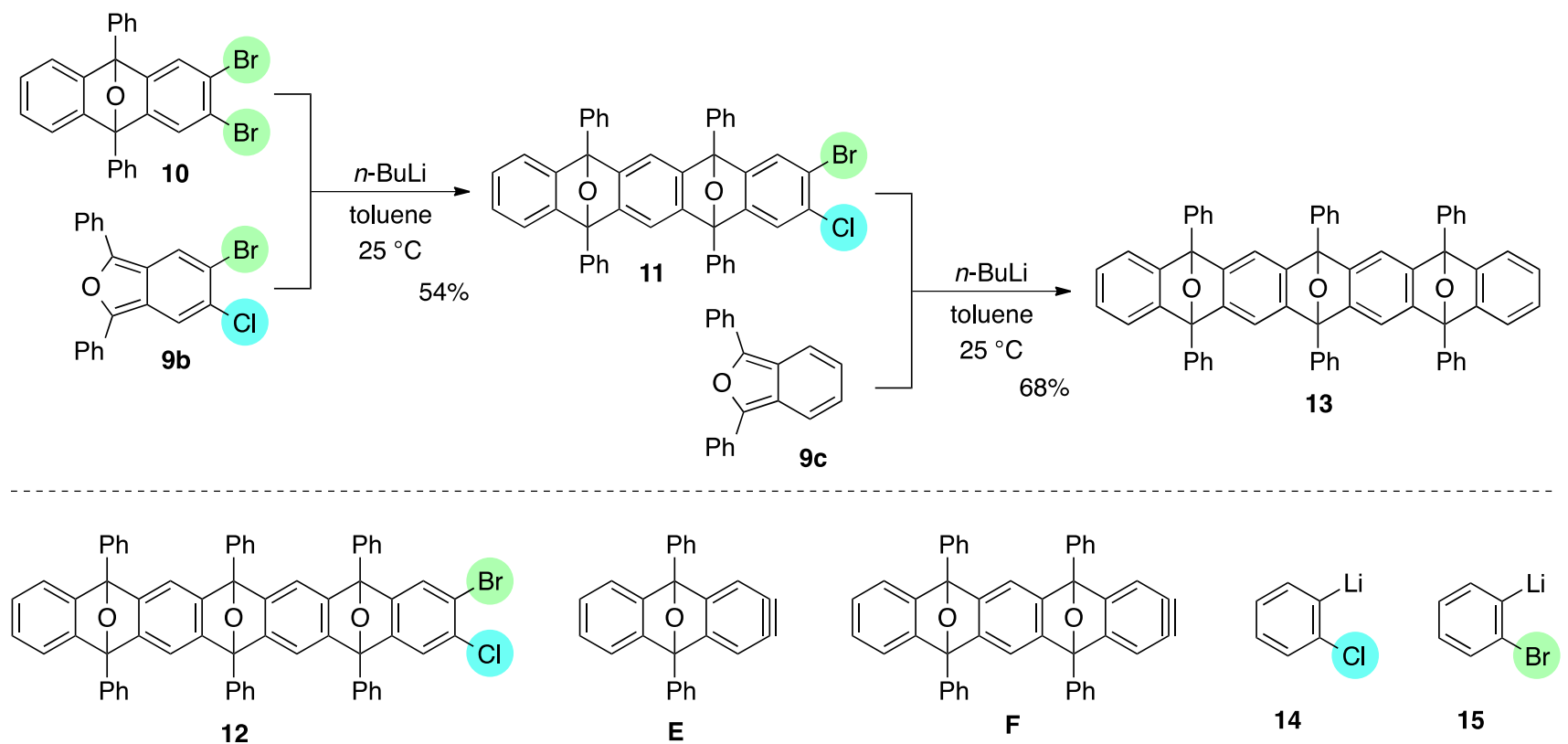

Scheme 5. Mono-directional [2+4] cycloadditions of arynes.

Moreover, it is worth mentioning that 1,2,4,5-tetrabromobenzene (16) nicely worked as a reactive platform [46-51], allowing bi-directional cycloadditions in an unsymmetrical manner (Scheme 6). The essential point of this sequential process is using 5-bromo-6-chloro-1,3-diphenylisobenzofuran (9b) to differentiate the reactivity of the two dihalogenated sites in the bis-aryne equivalent $\mathbf{1 7}$, which was efficiently obtained by the first [2+4] cycloaddition of dibromobenzyne $\mathbf{G}$ and isobenofuran $\mathbf{9 b}$. It is notable that perfect site-selectivity was observed in the bromine-lithium exchange of 16, selectively generating the dibromobenzyne $\mathbf{G}$ [52]. The cycloadduct 17, thus obtained, again underwent the selective bromine-lithium exchange at the dibromo side in 17, as a related reaction of dibromide $\mathbf{1 0}$ and isobenzofuran $\mathbf{9 b}$ (Scheme 5), generating the bromochlorobenzyne $\mathbf{H}$, which was intercepted by $\mathbf{9 c}$ to afford the unsymmetrical cycloadduct 11 in $65 \%$ yield, accompanied by a formation of dual cycloadduct $21(20 \%)$. Although the observed selectivity in the reaction of 17 was moderate $(\mathbf{1 1} / \mathbf{2 1}=3.2: 1)$, use of bis-aryne equivalent $\mathbf{1 7}$ with an unsymmetric form turned out to be indispensible to discriminate the reactivity of the two dihalogenated sites in $\mathbf{1 7}$, because the corresponding reaction of the symmetrical tetrabromide $\mathbf{2 0}$ resulted in the decreased selectivity in the formation of the desired mono-cycloadduct 21 and bis-cycloadduct $13(21 / 13=1.5: 1)$. Final [2+4] cycloaddition of aryne $\mathbf{F}$, generated from the bis-cycloadduct 11, with furan 18 under the above-mentioned conditions was satisfied, efficiently affording the tris-cycloadduct $\mathbf{1 9}$ with a various synthetic opportunity for further introduction of fused rings and/or functionalization. 


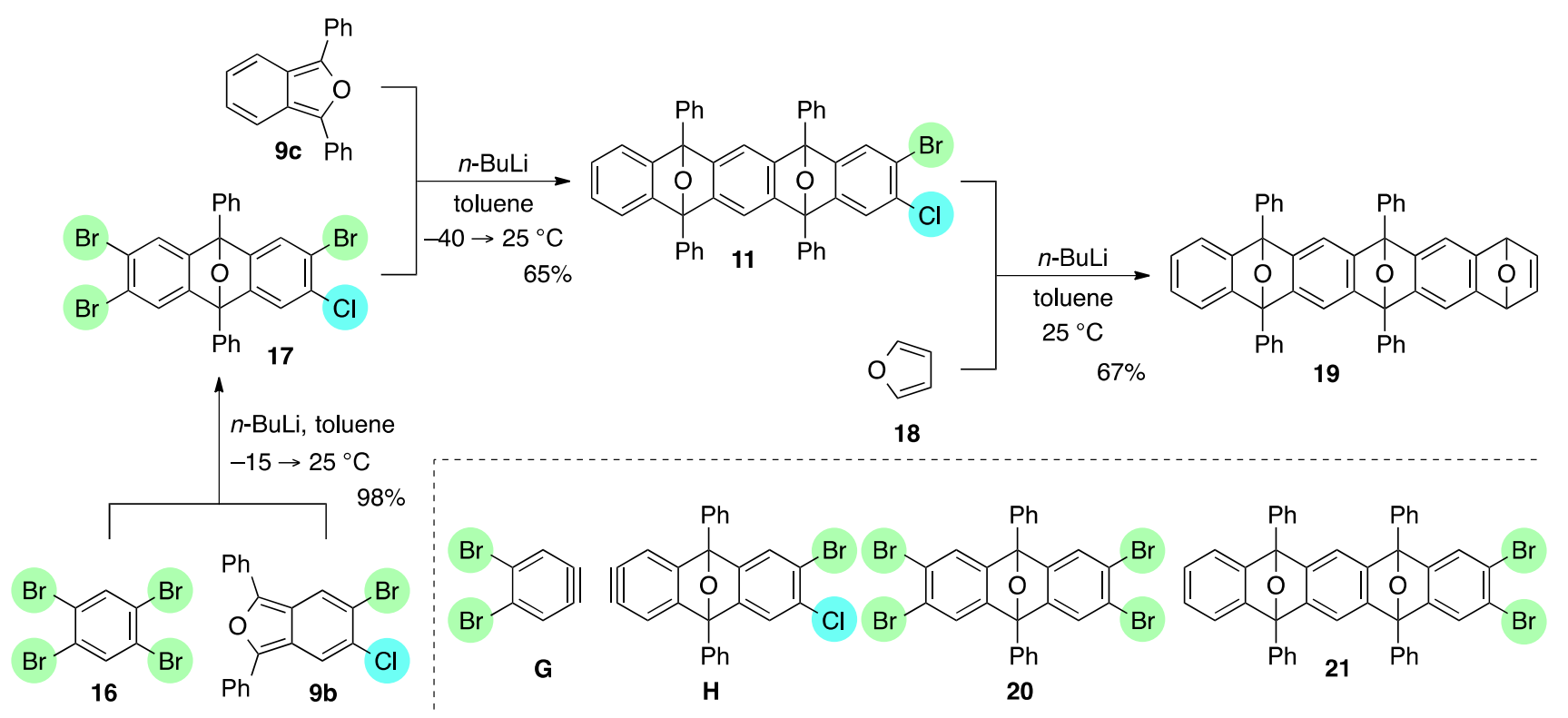

Scheme 6. Bi-directional [2+4] cycloadditions of arynes.

\section{Experimental Section}

\section{General Information}

All experiments dealing with air- and moisture-sensitive compounds were conducted under an atmosphere of dry argon. Toluene (anhydrous; Wako Pure Chemical Industries, Ltd., Osaka, Japan) was used as received. For thin-layer chromatography (TLC) analysis, Merck pre-coated plates (silica gel 60 $\mathrm{F}_{254}$, Art 5715, $0.25 \mathrm{~mm}$, Merck Japan, Tokyo, Japan) were used. For flash column chromatography, silica gel $60 \mathrm{~N}$ (spherical, neutral, 63-210 $\mu \mathrm{m}$ ) from Kanto Chemical (Tokyo, Japan) was used. Silica gel preparative TLC (PTLC) was performed on Merck silica gel $60 \mathrm{PF}_{254}$ (Art 7747).

${ }^{1} \mathrm{H}-\mathrm{NMR}$ and ${ }^{13} \mathrm{C}-\mathrm{NMR}$ were measured on a JNM ECA-300 and a JNM ECX-500II spectrometer (JEOL, Tokyo, Japan). Attenuated Total Reflectance Fourier Transformation Infrared (ATR-FTIR) spectra were recorded on a FT/IR-4200 FT-IR Spectrometer (JASCO, Tokyo, Japan). High resolution mass spectra were obtained with a JEOL JMS 700 spectrometer and a JEOL AccuTOF LC-plus JMS-T100LP. Melting point ( $\mathrm{mp}$ ) determinations were performed by using a MP-S3 instrument (Yanako, Kyoto, Japan) or a MPA100 Automated Melting Point System (OptiMelt, Sunnyvale, CA, USA) and are uncorrected.

Typical Procedure for [2+4] Cycloadditions of Aryne and Isobenzofuran: Synthesis of 2,3-Dibromo-9,10dihydro-9, 10-epoxyanthracene (6a). To a mixture of 1-bromo-2-iodobenzene (8a, $70.0 \mathrm{mg}, 0.247 \mathrm{mmol})$ and isobenzofuran $1(71.8 \mathrm{mg}, 0.260 \mathrm{mmol})$ in toluene $(2.0 \mathrm{~mL})$ was added $n$-BuLi (1.60 $\mathrm{M}$ in $n$-hexane, $0.19 \mathrm{~mL}, 0.30 \mathrm{mmol}$ ) at $-15^{\circ} \mathrm{C}$, and the reaction was warmed up to $25^{\circ} \mathrm{C}$. After $5 \mathrm{~min}$, the reaction was stopped by adding water. The products were extracted with EtOAc $(\times 3)$, and the combined organic extracts were washed with brine, dried ( $\left.\mathrm{Na}_{2} \mathrm{SO}_{4}\right)$, and concentrated in vacuo. The residue was purified by PTLC (hexane/EtOAc $=8 / 2$ ) to give 2,3-dibromo-9,10-dihydro-9,10-epoxyanthracene (6a, $67.9 \mathrm{mg}$, 78.1\%) as a white solid and 2,3-dibromo-5,7,12,14-tetrahydro-5,14:7,12-diepoxypentacene (7a, $1.2 \mathrm{mg}$, $1.0 \%$, ds: $17 / 83$ ) as a mixture of diastereomers. 
<smiles>Brc1cc2c(cc1Br)C1OC2c2ccccc21</smiles>

Compound 6a: Mp 208.5-209.1 ${ }^{\circ} \mathrm{C}\left(\right.$ hexane/ $\left.\mathrm{CHCl}_{3}\right)$; ${ }^{1} \mathrm{H}-\mathrm{NMR}\left(\mathrm{CDCl}_{3}, \delta\right) 6.01(\mathrm{~s}, 2 \mathrm{H}), 7.06(\mathrm{dd}, 2 \mathrm{H}$, $\left.J_{1}=3.1 \mathrm{~Hz}, J_{2}=5.2 \mathrm{~Hz}\right), 7.33\left(\mathrm{dd}, 2 \mathrm{H}, J_{1}=3.1 \mathrm{~Hz}, J_{2}=5.2 \mathrm{~Hz}\right), 7.55(\mathrm{~s}, 2 \mathrm{H}) ;{ }^{13} \mathrm{C}-\mathrm{NMR}\left(\mathrm{CDCl}_{3}, \delta\right)$ 82.0, 120.7, 121.6, 125.7, 126.5, 146.9, 149.2; IR (ATR) 3027, 1569, 1459, 1259, 1085, 953, 832, $762 \mathrm{~cm}^{-1}$; ${ }^{-}$HRMS (FAB) $m / z 351.8925$ (351.8922 calcd for $\mathrm{C}_{14} \mathrm{H}_{8} \mathrm{Br}_{2} \mathrm{O}, \mathrm{M}^{+}$).

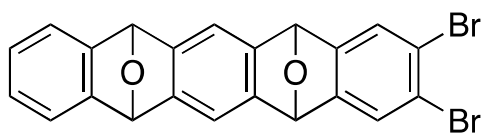

Compound 7a, less polar diastereomer: $\mathrm{R}_{f} 0.30$ (hexane/ $\mathrm{CH}_{2} \mathrm{Cl}_{2}=4 / 6$ ); $\mathrm{Mp}$ decomposed at $300{ }^{\circ} \mathrm{C}$; ${ }^{1} \mathrm{H}-\mathrm{NMR}\left(\mathrm{CDCl}_{3}, \delta\right) 5.90(\mathrm{~s}, 2 \mathrm{H}), 5.95(\mathrm{~s}, 2 \mathrm{H}), 7.00\left(\mathrm{dd}, 2 \mathrm{H}, J_{1}=3.1 \mathrm{~Hz}, J_{2}=5.5 \mathrm{~Hz}\right), 7.29(\mathrm{dd}, 2 \mathrm{H}$, $\left.J_{1}=3.1 \mathrm{~Hz}, J_{2}=5.5 \mathrm{~Hz}\right), 7.31(\mathrm{~s}, 2 \mathrm{H}), 7.53(\mathrm{~s}, 2 \mathrm{H}) ;{ }^{13} \mathrm{C}-\mathrm{NMR}\left(\mathrm{CDCl}_{3}, \delta\right) 81.9,82.4,114.2,120.4,121.6$, 125.6, 126.0, 146.1, 147.8, 148.1, 149.2; IR (ATR) 3016, 1569, 1457, 1265, 1085, 949, 832, $772 \mathrm{~cm}^{-1}$; ${ }^{-}$HRMS (FAB) $m / z 468.9262$ (468.9263 calcd for $\mathrm{C}_{22} \mathrm{H}_{13} \mathrm{Br}_{2} \mathrm{O}_{2},[\mathrm{M}+\mathrm{H}]^{+}$).

Compound 7a, more polar diastereomer: $\mathrm{R}_{f} 0.13$ (hexane $/ \mathrm{CH}_{2} \mathrm{Cl}_{2}=4 / 6$ ); $\mathrm{Mp}$ decomposed at $300{ }^{\circ} \mathrm{C}$; ${ }^{1} \mathrm{H}-\mathrm{NMR}\left(\mathrm{CDCl}_{3}, \delta\right) 5.90(\mathrm{~s}, 2 \mathrm{H}), 5.96(\mathrm{~s}, 2 \mathrm{H}), 6.97\left(\mathrm{dd}, 2 \mathrm{H}, J_{1}=3.1 \mathrm{~Hz}, J_{2}=5.2 \mathrm{~Hz}\right), 7.27(\mathrm{dd}, 2 \mathrm{H}$, $\left.J_{1}=3.1 \mathrm{~Hz}, J_{2}=5.2 \mathrm{~Hz}\right), 7.29(\mathrm{~s}, 2 \mathrm{H}), 7.46(\mathrm{~s}, 2 \mathrm{H}) ;{ }^{13} \mathrm{C}-\mathrm{NMR}\left(\mathrm{CDCl}_{3}, \delta\right) 82.0,82.5,113.9,120.5,121.5$, 125.6, 125.9, 146.0, 147.9, 149.2; IR (ATR) 3010, 1573, 1457, 1271, 1086, 952, 836, $754 \mathrm{~cm}^{-1}$; ${ }^{-} \mathrm{HRMS}$ (FAB) $m / z 468.9256\left(468.9263\right.$ calcd for $\left.\mathrm{C}_{22} \mathrm{H}_{13} \mathrm{Br}_{2} \mathrm{O}_{2},[\mathrm{M}+\mathrm{H}]^{+}\right)$.

2,3-Dibromo-9,10-diphenyl-9,10-epoxyanthracene (10). According to the procedure described for the synthesis of cycloadduct 6a, 1-bromo-2-iodobenzene (8a, $112 \mathrm{mg}, 0.396 \mathrm{mmol})$, isobenzofuran 9a (129 mg, $0.301 \mathrm{mmol}$ ) and $n$-BuLi (1.60 M in $n$-hexane, $0.25 \mathrm{~mL}, 0.40 \mathrm{mmol}$ ) gave, after purified by silica-gel flash column chromatography (hexane $\left./ \mathrm{CH}_{2} \mathrm{Cl}_{2} / \mathrm{Et}_{2} \mathrm{O}=98 / 1 / 1 \rightarrow 96 / 3 / 1\right)$, cycloadduct $10(110 \mathrm{mg}, 72.4 \%$ ) as a white solid.<smiles>Brc1cc2c(cc1Br)C1(c3ccccc3)OC2(c2ccccc2)c2ccccc21</smiles>

Compound 10: Mp 167.6-168.5 ${ }^{\circ} \mathrm{C}$ (hexane/Et $\left.2 \mathrm{O}\right)$; ${ }^{1} \mathrm{H}-\mathrm{NMR}\left(\mathrm{CDCl}_{3}, \delta\right) 7.08\left(\mathrm{dd}, 2 \mathrm{H}, J_{1}=2.9 \mathrm{~Hz}\right.$, $\left.J_{2}=5.7 \mathrm{~Hz}\right), 7.38\left(\mathrm{dd}, 2 \mathrm{H}, J_{1}=2.9 \mathrm{~Hz}, J_{2}=5.7 \mathrm{~Hz}\right), 7.49-7.53(\mathrm{~m}, 2 \mathrm{H}), 7.54(\mathrm{~s}, 2 \mathrm{H}), 7.59-7.63(\mathrm{~m}, 4 \mathrm{H})$, 7.86-7.89 (m, 4H); ${ }^{13} \mathrm{C}-\mathrm{NMR}\left(\mathrm{CDCl}_{3}, \delta\right) 90.2,120.7,121.8,125.6,126.4,126.5,128.7,129.0,133.9$, 149.2, 151.6; IR (ATR) 3030, 1599, 1499, 1295, 1036, 992, 871, $741 \mathrm{~cm}^{-1}$; ${ }^{-}$HRMS (DART) $\mathrm{m} / z$ 502.9644 (502.9646 calcd for $\mathrm{C}_{26} \mathrm{H}_{17} \mathrm{Br}_{2} \mathrm{O},[\mathrm{M}+\mathrm{H}]^{+}$).

2-Bromo-3-chloro-5, 7,12,14-tetraphenyl-5,14:7,12-diepoxypentacene (11). According to the procedure described for the synthesis of cycloadduct 6a, cycloadduct $10(75.6 \mathrm{mg}, 0.150 \mathrm{mmol})$, isobenzofuran $\mathbf{9 b}$ $(63.2 \mathrm{mg}, 0.165 \mathrm{mmol})$ and $n$-BuLi $(1.60 \mathrm{M}$ in $n$-hexane, $0.12 \mathrm{~mL}, 0.19 \mathrm{mmol})$ gave, after purification 
by silica-gel flash column chromatography (hexane $/ \mathrm{CH}_{2} \mathrm{Cl}_{2} / \mathrm{Et}_{2} \mathrm{O}=96 / 3 / 1 \rightarrow 88 / 9 / 3$ ), 2-bromo-3-chloro5,7,12,14-tetraphenyl-5,14:7,12-diepoxypentacene $(\mathbf{1 1}, 58.8 \mathrm{mg}, 53.9 \%$, ds. less polar/more polar $=$ 46/54) and 2-bromo-3-chloro-5,7,12,14-tetraphenyl-5,14:7,12-diepoxy-pentacene (12, $23.7 \mathrm{mg}, 15.9 \%)$ as a mixture of diastereomers, respectively. The diastereomers of $\mathbf{1 1}$ were separated by PTLC (hexane/toluene/ $\mathrm{CH}_{2} \mathrm{Cl}_{2} / \mathrm{Et}_{2} \mathrm{O}=82 / 10 / 6 / 2 \mathrm{X} 2$ ), affording less polar 11 and more polar 11 as white solids.

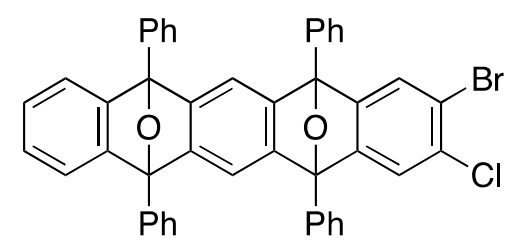

Compound 11, less polar: $\mathrm{R}_{f} 0.38$ (hexane/toluene/ $\mathrm{CH}_{2} \mathrm{Cl}_{2} / \mathrm{Et}_{2} \mathrm{O}=82 / 10 / 6 / 2, \mathrm{X} 2$ ); $\mathrm{Mp}$ decomposed at $240{ }^{\circ} \mathrm{C}\left(\mathrm{MeOH}_{\mathrm{CHCl}}\right) ;{ }^{1} \mathrm{H}-\mathrm{NMR}\left(\mathrm{CDCl}_{3}, \delta\right) 6.96\left(\mathrm{dd}, 2 \mathrm{H}, J_{1}=2.9 \mathrm{~Hz}, J_{2}=5.2 \mathrm{~Hz}\right), 7.27(\mathrm{dd}, 2 \mathrm{H}$, $\left.J_{1}=2.9 \mathrm{~Hz}, J_{2}=5.2 \mathrm{~Hz}\right), 7.29(\mathrm{~s}, 1 \mathrm{H}), 7.33(\mathrm{~s}, 2 \mathrm{H}), 7.44(\mathrm{~s}, 1 \mathrm{H}), 7.46-7.51(\mathrm{~m}, 4 \mathrm{H}), 7.56-7.60(\mathrm{~m}, 8 \mathrm{H})$, 7.77-7.79 (m, 4H), 7.83-7.86 (m, 4H); ${ }^{13} \mathrm{C}-\mathrm{NMR}\left(\mathrm{CDCl}_{3}, \delta\right)$ 90.2, 90.3, 90.5, 113.7, 119.3, 120.4, 122.6, 125.6, 125.8, 126.4, 126.6, 128.4, 128.7, 128.9, 129.1, 131.5, 133.70, 133.73, 134.6, 148.26, 148.34, 149.9, 150.15, 150.17, 150.6, 151.3; IR (ATR) 3059, 1607, 1500, 1308, 1083, 986, 867, $744 \mathrm{~cm}^{-1}$; ${ }^{-} \mathrm{HRMS}$ (ESI) $m / z 749.0834$ (749.0859 calcd for $\mathrm{C}_{46} \mathrm{H}_{28} \mathrm{BrClNaO}_{2},[\mathrm{M}+\mathrm{Na}]^{+}$).

Compound 11, more polar: $\mathrm{R}_{f} 0.28$ (hexane/toluene $/ \mathrm{CH}_{2} \mathrm{Cl}_{2} / \mathrm{Et}_{2} \mathrm{O}=82 / 10 / 6 / 2, \mathrm{X} 2$ ); $\mathrm{Mp}$ decomposed at $230{ }^{\circ} \mathrm{C}\left(\mathrm{MeOH}_{\mathrm{CHCl}}\right) ;{ }^{1} \mathrm{H}-\mathrm{NMR}\left(\mathrm{CDCl}_{3}, \delta\right) 7.02\left(\mathrm{dd}, 2 \mathrm{H}, J_{1}=2.9 \mathrm{~Hz}, J_{2}=5.2 \mathrm{~Hz}\right), 7.30(\mathrm{~s}, 2 \mathrm{H})$, 7.32-7.35 (m, 3H), 7.43-7.49 (m, 5H), 7.52-7.57 (m, 8H), 7.75-7.77 (m, 4H), 7.81-7.84 (m, 4H); ${ }^{13} \mathrm{C}-\mathrm{NMR}\left(\mathrm{CDCl}_{3}, \delta\right)$ 90.2, 90.3, 90.5, 113.49, 113.51, 119.3, 120.5, 122.7, 125.6, 125.7, 126.4, 126.5, 128.3, 128.7, 128.8, 129.0, 131.5, 133.7, 133.8, 134.6, 148.2 , 148.3, 149.9, 150.0, 150.3, 150.8, 151.6; IR (ATR) 3065, 1607, 1498, 1311, 1082, 989, 863, $746 \mathrm{~cm}^{-1}$; ${ }^{-}$HRMS (ESI) $m / z 749.0876$ (749.0859 calcd for $\left.\mathrm{C}_{46} \mathrm{H}_{28} \mathrm{BrClNaO}_{2},[\mathrm{M}+\mathrm{Na}]^{+}\right)$.

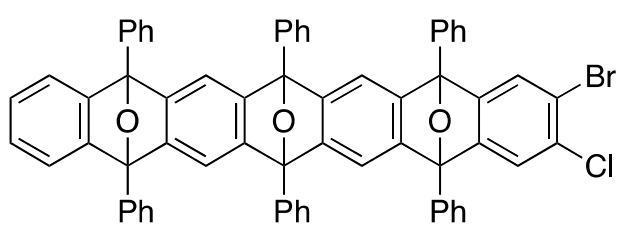

Compound 12 (a mixture of four diastereomers): ${ }^{1} \mathrm{H}-\mathrm{NMR}\left(\mathrm{CDCl}_{3}, \delta\right)$ 6.90-7.01 (m, 8H), 7.19-7.33 (m, 24H), 7.36-7.60 (m, 80H), 7.66-7.83 (m, 48H); ${ }^{13} \mathrm{C}-\mathrm{NMR}\left(\mathrm{CDCl}_{3}, \delta\right)$ 90.0, 90.05, 90.07, 90.09, 90.11, 90.14, 90.17, 90.19, 90.36, 90.39, 90.46, 90.50, 113.3, 113.4, 113.47, 113.51, 113.7, 113.8, 119.2, 119.3, $119.5,119.6,120.08,120.11,120.2,120.4,122.4,122.5,122.7,125.4,125.5,125.55,125.62,125.7$, $125.8,125.97,126.00,126.07,126.12,126.2,126.3,126.42,126.44,126.5,126.57,126.64,126.7$, $128.16,128.21,128.3,128.4,128.5,128.59,128.63,128.66,128.72,128.8,128.86,128.90,128.92$, $129.00,129.03,131.4,131.5,131.58,131.63,133.68,133.72,133.76,133.83,133.87,133.90,134.3$, $134.38,134.44,134.70,134.73,134.78,134.80,147.99,148.03,148.1,148.2,148.26,148.30,148.4$, $148.9,148.98,149.02,149.1,149.2,149.4,149.49,149.52,149.70,149.73,149.78,149.80,149.86$, 149.94, 149.96, 150.02, 150.2, 150.3, 150.35, 150.42, 150.5, 150.6, 151.18, 151.24, 151.37, 151.39; IR (ATR) 3062, 1606, 1499, 1307, 1082, 983, 885, $748 \mathrm{~cm}^{-1}$; ${ }^{-}$HRMS (ESI) $\mathrm{m} / z$ 1017.1756 (1017.1747 calcd for $\mathrm{C}_{66} \mathrm{H}_{40} \mathrm{BrClNaO}_{3},[\mathrm{M}+\mathrm{Na}]^{+}$). 
5,7,9,14,16,18-Hexaphenyl-5,18:7,16:9,14-triepoxyheptacene (13). According to the procedure described for the synthesis of cycloadduct 6a, cycloadduct 11 (more polar) ( $35.1 \mathrm{mg}, 0.0482 \mathrm{mmol}$ ), isobenzofuran 9c (14.7 mg, $0.0544 \mathrm{mmol})$ and $n$-BuLi (1.60 M in $n$-hexane, $0.040 \mathrm{~mL}, 0.064 \mathrm{mmol}$ ) gave, after purification by silica-gel flash column chromatography (hexane $/ \mathrm{CH}_{2} \mathrm{Cl}_{2} / \mathrm{Et}_{2} \mathrm{O}=96 / 3 / 1 \rightarrow 88 / 9 / 3$ ), 5,7,9,14,16,18hexaphenyl-5,18:7,16:9,14-triepoxyheptacene (13) as a mixture of diastereomers $(29.0 \mathrm{mg}, 68.0 \%$, ds. less polar/more polar $=46 / 54$ ). Those diastereomers were separated by PTLC (hexane/toluene $/ \mathrm{CH}_{2} \mathrm{Cl}_{2} / \mathrm{Et}_{2} \mathrm{O}=$ 78/10/8/4, X4), affording less polar 13 and more polar 13 as white solids, respectively.

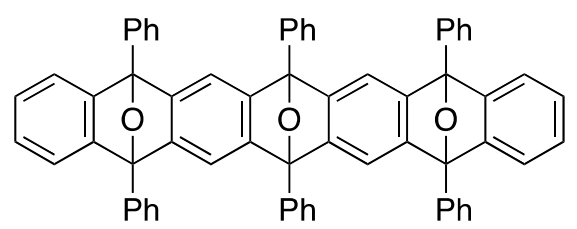

Compound 13 (less polar): $\mathrm{R}_{f} 0.55$ (hexane/toluene/ $\mathrm{CH}_{2} \mathrm{Cl}_{2} / \mathrm{Et}_{2} \mathrm{O}=78 / 10 / 8 / 4, \mathrm{X} 4$ ); $\mathrm{Mp}$ decomposed at $260{ }^{\circ} \mathrm{C}\left(\mathrm{MeOH} \mathrm{CHCl}_{3}\right) ;{ }^{1} \mathrm{H}-\mathrm{NMR}\left(\mathrm{CDCl}_{3}, \delta\right) ; 6.92\left(\mathrm{dd}, 4 \mathrm{H}, J_{1}=2.9 \mathrm{~Hz}, J_{2}=5.2 \mathrm{~Hz}\right), 7.22(\mathrm{~s}, 4 \mathrm{H}), 7.23$ $\left(\mathrm{dd}, 4 \mathrm{H}, J_{1}=2.9 \mathrm{~Hz}, J_{2}=5.2 \mathrm{~Hz}\right), 7.44-7.49(\mathrm{~m}, 6 \mathrm{H}), 7.52-7.56(\mathrm{~m}, 12 \mathrm{H}), 7.72-7.75(\mathrm{~m}, 4 \mathrm{H}), 7.79-7.82$ (m, 8H); ${ }^{13} \mathrm{C}-\mathrm{NMR}\left(\mathrm{CDCl}_{3}, \delta\right)$ 90.4, 113.4, 120.3, 125.6, 126.5, 126.6, 128.2, 128.3, 128.8, 128.9, 134.6, 134.8, 149.2, 149.4, 150.1; IR (ATR) 3058, 1603, 1496, 1307, 974, 867, $747 \mathrm{~cm}^{-1}$; ${ }^{-}$HRMS (ESI) $\mathrm{m} / \mathrm{z}$ 905.3020 (905.3032 calcd for $\mathrm{C}_{66} \mathrm{H}_{42} \mathrm{NaO}_{3},[\mathrm{M}+\mathrm{Na}]^{+}$).

Compound 13 (more polar): $\mathrm{R}_{f} 0.49$ (hexane/toluene/ $\mathrm{CH}_{2} \mathrm{Cl}_{2} / \mathrm{Et}_{2} \mathrm{O}=78 / 10 / 8 / 4, \mathrm{X} 4$ ); $\mathrm{Mp}$ decomposed at $250{ }^{\circ} \mathrm{C}\left(\mathrm{MeOH}_{\mathrm{CHCl}}\right) ;{ }^{1} \mathrm{H}-\mathrm{NMR}\left(\mathrm{CDCl}_{3}, \delta\right) 6.94\left(\mathrm{dd}, 2 \mathrm{H}, J_{1}=2.9 \mathrm{~Hz}, J_{2}=5.2 \mathrm{~Hz}\right), 6.96(\mathrm{dd}, 2 \mathrm{H}$, $\left.J_{1}=2.9 \mathrm{~Hz}, J_{2}=5.2 \mathrm{~Hz}\right), 7.19(\mathrm{~s}, 2 \mathrm{H}), 7.22-7.25(\mathrm{~m}, 4 \mathrm{H}), 7.28(\mathrm{~s}, 2 \mathrm{H}), 7.39-7.59(\mathrm{~m}, 18 \mathrm{H}), 7.72-7.74$ (m, 4H), 7.77-7.80 (m, 4H), 7.81-7.83 (m, 4H); ${ }^{13} \mathrm{C}-\mathrm{NMR}\left(\mathrm{CDCl}_{3}, \delta\right)$ 90.37, 90.39, 90.5, 113.2, 113.5, 120.2 , 120.3, 125.7, 125.8, 126.4, 126.5, 126.6, 128.11, 128.13, 128.3, 128.6, 128.8, 134.5, 134.8, 134.9, 149.0, 149.1, 149.3, 149.6, 149.9, 150.1; IR (ATR) 3063, 1602, 1497, 1308, 977, 869, $747 \mathrm{~cm}^{-1}$; ${ }^{-} \mathrm{HRMS}$ (ESI) $m / z 905.3028\left(905.3032\right.$ calcd for $\left.\mathrm{C}_{66} \mathrm{H}_{42} \mathrm{NaO}_{3},[\mathrm{M}+\mathrm{Na}]^{+}\right)$.

2,3,6-Tribromo-7-chloro-9,10-diphenyl-9,10-epoxyanthracene (17). According to the procedure described for the synthesis of cycloadduct 6a, 1,2,4,5-tetrabromobenzene (16, $1.54 \mathrm{~g}, 3.91 \mathrm{mmol})$, isobenzofuran 9b $(1.00 \mathrm{~g}, 2.61 \mathrm{mmol})$ and $n$-BuLi (1.60 M in $n$-hexane, $2.50 \mathrm{~mL}, 4.00 \mathrm{mmol})$ gave, after purification by silica-gel flash column chromatography (hexane/ $\mathrm{CH}_{2} \mathrm{Cl}_{2} / \mathrm{Et}_{2} \mathrm{O}=96 / 3 / 1$ ), 2,3,6-tribromo-7-chloro-9,10diphenyl-9,10-epoxyanthracene $(\mathbf{1 7}, 1.58 \mathrm{~g}, 98.1 \%)$ as a white solid.<smiles>Clc1cc2c(cc1Br)C1(c3ccccc3)COC2(c2ccccc2)c2cc(Br)c(Br)cc21</smiles>

Compound 17: Mp 247.2-248.0 ${ }^{\circ} \mathrm{C}$ (hexane/ $\left.\mathrm{CHCl}_{3}\right) ;{ }^{1} \mathrm{H}-\mathrm{NMR}\left(\mathrm{CDCl}_{3}, \delta\right) 7.41(\mathrm{~s}, 1 \mathrm{H}), 7.52-7.56$ $(\mathrm{m}, 2 \mathrm{H}), 7.559(\mathrm{~s}, 1 \mathrm{H}), 7.564(\mathrm{~s}, 2 \mathrm{H}), 7.61-7.65(\mathrm{~m}, 4 \mathrm{H}), 7.80-7.83(\mathrm{~m}, 4 \mathrm{H}) ;{ }^{13} \mathrm{C}-\mathrm{NMR}\left(\mathrm{CDCl}_{3}, \delta\right) 89.8$, $90.0,120.1,122.5,122.9,125.9,126.3,129.1,129.2,132.3,132.89,132.92,149.5,150.3,150.37$, 150.44; IR (ATR) 3017, 1601, 1499, 1288, 1089, 987, 887, $746 \mathrm{~cm}^{-1}$; ${ }^{-}$HRMS (DART) $\mathrm{m} / z 614.8381$ (614.8362 calcd for $\mathrm{C}_{26} \mathrm{H}_{15} \mathrm{Br}_{3} \mathrm{ClO},[\mathrm{M}+\mathrm{H}]^{+}$). 
2-Bromo-3-chloro-5, 7,12,14-tetraphenyl-5,14:7,12-diepoxypentacene (11). According to the procedure described for the synthesis of cycloadduct 6a, cycloadduct $17(124 \mathrm{mg}, 0.201 \mathrm{mmol})$, isobenzofuran 9c $(59.7 \mathrm{mg}, 0.221 \mathrm{mmol})$ and $n$-BuLi $(1.60 \mathrm{M}$ in $n$-hexane, $0.15 \mathrm{~mL}, 0.24 \mathrm{mmol})$ gave, after purification by silica-gel flash column chromatography (hexane $/ \mathrm{CH}_{2} \mathrm{Cl}_{2} / \mathrm{Et}_{2} \mathrm{O}=96 / 3 / 1 \rightarrow 88 / 9 / 3$ ), cycloadduct 11 $(94.3 \mathrm{mg}, 64.7 \%$, ds. less polar $/$ more polar $=52 / 48)$ and cycloadduct $\mathbf{1 3}$ as a mixture of diastereomers (33.6 mg, 20.0\%), respectively.

1,4-Dihydro-6,8,13,15-tetraphenyl-1,4:6,15:8,13-triepoxyhexacene (19). According to the procedure described for the synthesis of cycloadduct 6a, cycloadduct 11 (less polar) (67.9 mg, $0.0933 \mathrm{mmol}$ ), furan 18 (65 mg, $0.96 \mathrm{mmol}$ ) and $n$-BuLi (1.63 M in $n$-hexane, $0.075 \mathrm{~mL}, 0.12 \mathrm{mmol}$ ) gave, after purification by PTLC (hexane $/ \mathrm{CH}_{2} \mathrm{Cl}_{2}$ /acetone = 7/2/1), 1,4-dihydro-6,8,13,15-tetraphenyl-1,4:6,15:8,13-triepoxyhexacene (19) as a mixture of diastereomers $(42.3 \mathrm{mg}, 66.6 \%)$.

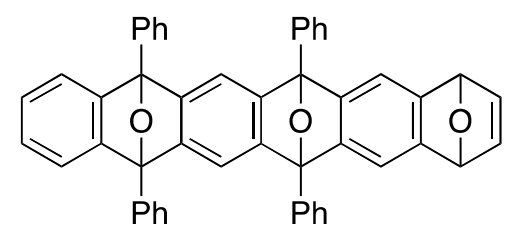

Compound 19 (a mixture of two diastereomers): ${ }^{1} \mathrm{H}-\mathrm{NMR}\left(\mathrm{CDCl}_{3}, \delta\right) 5.50(\mathrm{~s}, 2 \mathrm{H}), 5.52(\mathrm{~s}, 2 \mathrm{H}), 6.88$ (s, 2H), $6.91(\mathrm{~s}, 2 \mathrm{H}), 6.92-6.95(\mathrm{~m}, 4 \mathrm{H}), 7.13(\mathrm{~s}, 2 \mathrm{H}), 7.19(\mathrm{~s}, 2 \mathrm{H}), 7.23\left(\mathrm{dd}, 2 \mathrm{H}, J_{1}=2.9 \mathrm{~Hz}, J_{2}=5.2 \mathrm{~Hz}\right)$, $7.26\left(\mathrm{dd}, 2 \mathrm{H}, J_{1}=2.9 \mathrm{~Hz}, J_{2}=5.2 \mathrm{~Hz}\right), 7.29(\mathrm{~s}, 2 \mathrm{H}), 7.33(\mathrm{~s}, 2 \mathrm{H}), 7.44-7.49(\mathrm{~m}, 8 \mathrm{H}), 7.54-7.59(\mathrm{~m}, 16 \mathrm{H})$, 7.79-7.87 (m, 16H); ${ }^{13} \mathrm{C}-\mathrm{NMR}\left(\mathrm{CDCl}_{3}, \delta\right)$ 82.2, 82.3, 90.47, 90.52, 113.40, 113.43, 113.6, 113.8, 120.2, $120.4,125.6,125.7,126.5,126.55,126.60,126.9,128.3,128.76,128.81,134.8,134.9,143.19,143.22$, 148.2, 148.3, 148.6, 148.8, 149.2, 149.5, 149.8, 150.0, 150.2; IR (ATR) 3062, 1602, 1499, 1308, 984, 848, 744, $700 \mathrm{~cm}^{-1}$; ${ }^{-}$HRMS (ESI) $m / z 703.2233$ (703.2249 calcd for $\mathrm{C}_{50} \mathrm{H}_{32} \mathrm{NaO}_{3},[\mathrm{M}+\mathrm{Na}]^{+}$).

2,3-Dibromo-5, 7,12,14-tetraphenyl-5,14:7,12-diepoxypentacene (21). According to the procedure described for the synthesis of cycloadduct 6a, 2,3,6,7-tetrabromo-9,10-diphenyl-9,10-epoxyanthracene (20, $110 \mathrm{mg}$, $0.166 \mathrm{mmol})$ and isobenzofuran $9 \mathrm{c}(49.5 \mathrm{mg}, 0.183 \mathrm{mmol})$ and $n$-BuLi (1.63 $\mathrm{M}$ in $n$-hexane, $0.12 \mathrm{~mL}$, $0.20 \mathrm{mmol}$ ) gave, after purification by silica-gel flash column chromatography (hexane $/ \mathrm{CH}_{2} \mathrm{Cl}_{2} / \mathrm{Et}_{2} \mathrm{O}=$ 96/3/1 $\rightarrow 88 / 9 / 3)$, 2,3-dibromo-5, 7,12,14-tetraphenyl-5,14:7,12-diepoxypentacene (21, $45.4 \mathrm{mg}, 35.4 \%$, ds. less polar/more polar $=48 / 52)$ and 2,3-dibromo-5,7,12,14-tetraphenyl-5,14:7,12-diepoxypentacene $(\mathbf{1 3})$ as a mixture of diastereomers $(35.0 \mathrm{mg}, 23.9 \%)$, respectively.<smiles>Brc1cc2c(cc1Br)C1(c3ccccc3)OC2(c2ccccc2)c2cc3c(cc21)C1(c2ccccc2)OC3(c2ccccc2)c2ccccc21</smiles>

Compound 21 (less polar): $\mathrm{R}_{f} 0.62$ (hexane/toluene/ $\mathrm{CH}_{2} \mathrm{Cl}_{2} / \mathrm{Et}_{2} \mathrm{O}=82 / 10 / 6 / 2, \mathrm{X} 3$ ); $\mathrm{Mp}$ decomposed at $250{ }^{\circ} \mathrm{C}\left(\mathrm{MeOH}_{\mathrm{CHCl}}\right) ;{ }^{1} \mathrm{H}-\mathrm{NMR}\left(\mathrm{CDCl}_{3}, \delta\right) 6.96\left(\mathrm{dd}, 2 \mathrm{H}, J_{1}=2.9 \mathrm{~Hz}, J_{2}=5.2 \mathrm{~Hz}\right), 7.27(\mathrm{dd}, 2 \mathrm{H}$, $\left.J_{1}=2.9 \mathrm{~Hz}, J_{2}=5.2 \mathrm{~Hz}\right), 7.33(\mathrm{~s}, 2 \mathrm{H}), 7.44(\mathrm{~s}, 2 \mathrm{H}), 7.46-7.51(\mathrm{~m}, 4 \mathrm{H}), 7.56-7.60(\mathrm{~m}, 8 \mathrm{H}), 7.76-7.79$ (m, 4H), 7.83-7.86 (m, 4H); ${ }^{13} \mathrm{C}-\mathrm{NMR}\left(\mathrm{CDCl}_{3}, \delta\right)$ 90.2, 90.5, 113.7, 120.4, 121.7, 125.6, 125.8, 126.4, 
126.6, 128.4, 128.7, 128.9, 129.0, 133.7, 134.6, 148.3, 149.9, 150.2, 151.4; IR (ATR) 3059, 1606, 1499, 1308, 1033, 984, 866, $743 \mathrm{~cm}^{-1}$; ${ }^{-} \mathrm{HRMS}$ (ESI) $\mathrm{m} / \mathrm{z} 793.0333$ (793.0354 calcd for $\mathrm{C}_{46} \mathrm{H}_{28} \mathrm{Br}_{2} \mathrm{NaO}_{2}$, [M+ Na] $]^{+}$.

Compound 21 (more polar): $\mathrm{R}_{f} 0.52$ (hexane/toluene/ $\mathrm{CH}_{2} \mathrm{Cl}_{2} / \mathrm{Et}_{2} \mathrm{O}=82 / 10 / 6 / 2, \mathrm{X} 3$ ); $\mathrm{Mp}$ decomposed at $250{ }^{\circ} \mathrm{C}\left(\mathrm{MeOH} / \mathrm{CHCl}_{3}\right) ;{ }^{1} \mathrm{H}-\mathrm{NMR}\left(\mathrm{CDCl}_{3}, \delta\right) 7.01\left(\mathrm{dd}, 2 \mathrm{H}, J_{1}=2.9 \mathrm{~Hz}, J_{2}=5.2 \mathrm{~Hz}\right), 7.31(\mathrm{~s}, 2 \mathrm{H}), 7.33$ $\left(\mathrm{dd}, 2 \mathrm{H}, J_{1}=2.9 \mathrm{~Hz}, J_{2}=5.2 \mathrm{~Hz}\right), 7.42-7.48(\mathrm{~m}, 4 \mathrm{H}), 7.482(\mathrm{~s}, 2 \mathrm{H}), 7.52-7.57(\mathrm{~m}, 8 \mathrm{H}), 7.75-7.77$ $(\mathrm{m}, 4 \mathrm{H}), 7.81-7.84(\mathrm{~m}, 4 \mathrm{H}) ;{ }^{13} \mathrm{C}-\mathrm{NMR}\left(\mathrm{CDCl}_{3}, \delta\right) 90.2,90.5,113.5,120.5,121.7,125.70,125.74,126.4$, 126.6, 128.3, 128.7, 128.8, 129.0, 133.7, 134.7, 148.2, 150.0, 150.3, 151.7; IR (ATR) 3063, 1601, 1499, 1310, 1032, 983, 862, $745 \mathrm{~cm}^{-1}$; ${ }^{-} \mathrm{HRMS}$ (ESI) $\mathrm{m} / z$ 793.0361 (793.0354 calcd for $\mathrm{C}_{46} \mathrm{H}_{28} \mathrm{Br}_{2} \mathrm{NaO}_{2},[\mathrm{M}+\mathrm{Na}]^{+}$).

\section{Conclusions}

Site-selective halogen-lithium exchange of 1,2-dihaloarenes allowed for the successive generation of benzynes and subsequent multiple [2+4] cycloadditions with various arynophiles to give highly functionalized polycyclic compounds, which were amenable to selective transformation en route to substituted polyacene derivatives. Further synthetic applications are under active investigation in our laboratories.

\section{Supplementary Materials}

Supplementary materials can be accessed at: http://www.mdpi.com/1420-3049/20/10/19449/s1.

\section{Acknowledgments}

This work was partially supported by the Grant-in-Aid for Scientific Research on Innovative Areas 2707 Middle Molecular Strategy from MEXT, Japan, and ACT-C from the Japan Science and Technology Agency.

\section{Author Contributions}

S.E. performed the experiments; S.E. and T.H. designed the experiments and wrote the paper.

\section{Conflicts of Interest}

The authors declare no conflict of interest.

\section{References and Notes}

1. Hoffmann, R.W. Dehydrobenzene and Cycloalkynes; Academic: New York, NY, USA, 1967.

2. Kessar, S.V. Nucleophilic coupling with arynes. In Comprehensive Organic Synthesis; Trost, B.M., Ed.; Pergamon: Oxford, UK, 1991; Volume 4, pp. 483-515.

3. Pellissier, H.; Santelli, M. The use of arynes in organic synthesis. Tetrahedron 2003, 59, 701-730.

4. Wenk, H.H.; Winkler, M.; Sander, W. One century of aryne chemistry. Angew. Chem. Int. Ed. 2003, $42,502-528$. 
5. Yoshida, H.; Takaki, K. Aryne insertion reactions into carbon-carbon $\sigma$-bonds. Synlett, 2012, 23, $1725-1732$.

6. Bhunia, A.; Yetra, S.R.; Biju, A.T. Recent advances in transition-metal-free carbon-carbon and carbon-heteroatom bond-forming reactions using arynes. Chem. Soc. Rev. 2012, 41, 3140-3152.

7. Dubrovskiy, A.V.; Markina, N.A.; Larock, R.C. Use of benzynes for the synthesis of heterocycles. Org. Biomol. Chem. 2013, 11, 191-218.

8. Hamura, T.; Chuda, Y.; Nakatsuji, Y.; Suzuki, K. Catalytic generation of arynes and trapping by nucleophilic addition and iodination. Angew. Chem. Int. Ed. 2012, 51, 3368-3372.

9. Haneda, H.; Eda, S.; Aratani, M.; Hamura, T. Dibromoisobenzofuran as a formal equivalent of didehydroisobenzofuran: Reactive platform for expeditious assembly of polycycles. Org. Lett. 2014, 16, 286-289.

10. Friedrichsen, W. Benzo[c]furans. Adv. Heterocycl. Chem. 1980, 26, 135-241.

11. Friedrichsen, W. Recent advances in the chemistry of benzo[c]furans and related compounds. $A d v$. Heterocycl. Chem. 1999, 73, 1-96.

12. Binger, P.; Wedemann, P.; Goddard, R.; Brinker, U.H. Cyclopropene: A new simple synthesis and Diels-Alder reactions with cyclopentadiene and 1,3-diphenylisobenzofuran. J. Org. Chem. 1996, 61, 6462-6464.

13. Rodrigo, R. Progress in the chemistry of isobenzofurans: Applications to the synthesis of natural products and polyaromatic hydrocarbons. Tetrahedron 1988, 44, 2093-2135.

14. Wong, H.N.C. Synthesis of novel benzenoid molecules by low-valent-titanium deoxygenation. Acc. Chem. Res. 1989, 22, 145-152.

15. Man, Y.-M.; Mak, T.C.W.; Wong, H.N.C. Synthesis of benzo-fused tetraphenylenes and crystal structure of a 4:1 clathrate inclusion compound of dibenzo[b,h]tetraphenylene with $p$-xylene. J. Org. Chem. 1990, 55, 3214-3221.

16. Chan, S.-H.; Yick, C.-Y.; Wong, H.N.C. 5,6-Bis(trimethylsilyl)benzo[c]furan: An isolable versatile building block for linear polycyclic aromatic compounds. Tetrahedron 2002, 58, 9413-9422.

17. Rainbolt, J.E.; Miller, G.P. 4,7-Diphenylisobenzofuran: A useful intermediate for the construction of phenyl-substituted acenes. J. Org. Chem. 2007, 72, 3020-3030.

18. Warrener, R.N. The isolation of isobenzofuran, a stable but highly reactive molecule. J. Am. Chem. Soc. 1971, 93, 2346-2348.

19. Warrener, R.N.; Shang, M.; Butler, D.N. A new stabilised form of isobenzofuran, rack-mounted on an alicyclophane. Chem. Commun. 2001, 1550-1551, doi:10.1039/B104383H.

20. Sygula, A.; Sygula, R.; Rabideau, P.W. Isocorannulenofuran: A versatile building block for the synthesis of large buckybowls. Org. Lett. 2006, 8, 5909-5911.

21. Pei, B.J.; Chan, W.H.; Lee, A.W.M. Anthracene capped isobenzofuran: A synthon for the preparations of iptycenes and iptycene quinones. Org. Lett. 2011, 13, 1774-1777.

22. Hamura, T.; Nakayama, R. A one-pot preparation of 1,3-diarylisobenzofuran. Chem. Lett. 2013, 42, 1013-1015.

23. Asahina, K.; Matsuoka, S.; Nakayama, R.; Hamura, T. An efficient synthetic route to 1,3-bis(arylethynyl)isobenzofuran using alkoxybenzocyclobutenone as a reactive platform. Org. Biomol. Chem. 2014, 12, 9773-9776. 
24. Cao, Y.; Wang, Xiao Y.; Wang, J.-Y.; Pei, J. Iron(III) chloride promoted cyclization: A facile approach to polycyclic aromatics for functional materials. Synlett 2014, 25, 313-323.

25. Reyes, E.; Uria, U.; Carrillo, L.; Vicario, J.L. Transannular reactions in asymmetric total synthesis. Tetrahedron 2014, 70, 9461-9484.

26. Scott, L.T. Polycyclic aromatic hydrocarbon bowls, baskets, balls and tubes: Challenging targets for chemical synthesis. Polycycl. Aromat. Compd. 2010, 30, 247-259.

27. Clayden, J. Regioselective synthesis of organolithiums by X-Li exchange. Organolithiums: selectivity for synthesis. In Tetrahedron Organic Chemistry Series; Pergamon: Oxford, UK, 2002; Volume 23, pp. 111-147.

28. Beak, P.; Allen, D.J. Experimental evaluation of transition structure geometry for an aryl bromide-alkyllithium exchange reaction: New information relevant to the reaction mechanism. J. Am. Chem. Soc. 1992, 114, 3420-3425.

29. Nájera, C.; Sansano, J.M.; Yus, M. Recent synthetic uses of functionalised aromatic and heteroaromatic organolithium reagents prepared by non-deprotonating methods. Tetrahedron 2003, 59, 9255-9303.

30. Dabrowski, M.; Kubicka, J.; Lulinski, S.; Serwatowski, J. Halogen-lithium exchange between substituted dihalobenzenes and butyllithium: Application to the regioselective synthesis of functionalized bromobenzaldehydes. Tetrahedron 2005, 61, 6590-6595.

31. Eda, S.; Eguchi, F.; Haneda, H.; Hamura, T. A new synthetic route to substituted tetracenes and pentacenes via stereoselective [4+2] cycloadditions of 1,4-dihydro-1,4-epoxynaphthalene and isobenzofuran. Chem. Commun. 2015, 51, 5963-5966.

32. Akita, R.; Kawanishi, K.; Hamura, T. Ring selective generation of isobenzofuran for divergent access to polycyclic aromatic compounds. Org. Lett. 2015, 17, 3094-3097.

33. Gilman, H.; Gorsich, R.D. Some reactions of $o$-halobromobenzenes with $n$-butyllithium. J. Am. Chem. Soc. 1956, 78, 2217-2222.

34. Chen, L.S.; Chen, G.J.; Tamborski, C. The synthesis and reactins of ortho bromophenyllithium. J. Organomet. Chem. 1980, 193, 283-292.

35. Caster, K.C.; Keck, C.G.; Walls, R.D. Synthesis of benzonorbornadienes: Regioselective benzyne formation. J. Org. Chem. 2001, 66, 2932-2936.

36. Coe, J.W.; Wirtz, M.C.; Bashore, C.G.; Candler, J. Formation of 3-halobenzyne: Solvent effects and cycloaddition adducts. Org. Lett. 2004, 6, 1589-1592.

37. Nagaki, A.; Ichinari, D.; Yoshida, J. Three-component coupling based on flash chemistry. Carbolithiation of benzyne with functionalized aryllithiums followed by reactions with electrophiles. J. Am. Chem. Soc. 2014, 136, 12245-12248.

38. Voss, G.; Gerlach, H. Regioselektiver brom/lithium-austausch bei 2,5-dibromo-1-nitrobenzol. Eine einfache synthese von 4-brom-2-nitrobenzaldehyd und 6,6'-dibromoindigo. Chem. Ber. 1989, 122, 1199-1201.

39. Stanetty, P.; Krumpak, B.; Rodler, I.K. Regioselectivity in sequential bromine-lithium exchange reactions of 2,4-dibromo- $N, N$-dimethylaniline. J. Chem. Res. 1995, 9, 342-343.

40. For preparation, see Supporting Information.

41. Hamura, T.; Arisawa, T.; Matsumoto, T.; Suzuki, K. Two-directional annelation: Dual benzyne cycloadditions starting from bis(sulfonyloxy)diiodobenzene. Angew. Chem. Int. Ed. 2006, 45, 6842-6844. 
42. Bendikov, M.; Wudl, F.; Perepichka, D.F. Tetrathiafulvalenes, oligoacenenes, and their buckminsterfullerene derivatives: The brick and mortar of organic electronics. Chem. Rev. 2004, 104, 4891-4946.

43. Anthony, J.E. Functionalized acenes and heteroacenes for organic electronics. Chem. Rev. 2006, 106, 5028-5048.

44. Anthony, J.E. The larger acenes: Versatile organic semiconductors. Angew. Chem. Int. Ed. 2008, 47, 452-483.

45. Bettinger, H.F. Electronic structure of higher acenes and polyacene: The perspective developed by theoretical analyses. Pure Appl. Chem. 2010, 82, 905-915.

46. Hart, H.; Ok, D. Synthesis of 1,5-diamino-1,5-dihydrobenzo[1,2-d:4,5-d']bistriazole (DABT) and its use as a 1,4-benzadiyne equivalent. J. Org. Chem. 1986, 51, 979-986.

47. Hart, H.; Lai, C.-Y.; Nwokogu, G.; Shamouilian, S.; Teuerstein, A.; Zlotogorski, C. Bisannelation of arenes with bis-aryne equivalents. J. Am. Chem. Soc. 1980, 102, 6649-6651.

48. Chen, Y.-L.; Sun, J.-Q.; Wei, X.; Wong, W.-W.; Lee, A.W.M. Generation of synthetic equivalents of benzdiynes from benzobisoxadisiloles. J. Org. Chem. 2004, 69, 7190-7197.

49. Morton, G.E.; Barrett, A.G.M. 1,4-Difluoro-2,5-dimethoxybenzene as a precursor for iterative double benzyne-furan Diels-Alder reactions. J. Org. Chem. 2005, 70, 3525-3529.

50. Schuster, I.I.; Cracium, L.; Ho, D.M.; Pascal, R.A., Jr. Synthesis of a strained, air-sensitive, polycyclic aromatic hydrocarbon by means of a new 1,4-benzadiyne equivalent. Tetrahedron $\mathbf{2 0 0 2}$, $58,8875-8882$.

51. Duong, H.M.; Bendikov, M.; Steiger, D.; Zhang, Q.; Sonmez, G.; Yamada, J.; Wudl, F. Efficient synthesis of a novel, twisted and stable, electroluminescent "twistacene". Org. Lett. 2003, 5, 4433-4436.

52. This site selectivity in the bromine-lithium exchange is due to the electron withdrawal of the four bromo atoms in 16, which leads to the selective generation of tribromophenyllithium. Subsequent elimination of lithium bromide from this intermediate produced dibromobenzyne $\mathbf{G}$.

Sample Availability: Not available.

(C) 2015 by the authors; licensee MDPI, Basel, Switzerland. This article is an open access article distributed under the terms and conditions of the Creative Commons Attribution license (http://creativecommons.org/licenses/by/4.0/). 